\title{
Les macrophages du cerveau humain : de la coloration argentique au SIDA
}

Les macrophages représentent de 20 à $30 \%$ des cellules gliales incluant la microglie résidente, la microglie périvasculaire et les monocytes. Ces cellules sont avant tout responsables de la réponse immune intracérébrale, mais pourraient aussi intervenir dans l'ontogenèse du cerveau. Dans les atteintes cérébrales du SIDA, les macrophages, et plus particulièrement les monocytes ou les cellules de la microglie périvasculaire, sont les cibles privilégiées de l'infection virale. Les désordres neurologiques induits pourraient résulter d'une action à distance ou d'un contact direct entre les macrophages infectés et les cellules neuronales ou macrogliales, et être relayés par des protéines virales ou par des facteurs cellulaires encore mal caractérisés. Dans d'autres maladies cérébrales, la sécrétion exagérée de cytokines par les cellules de la microglie pourrait jouer un rôle pathogénique.

\section{Marc Tardieu Sylviane Peudenier Guillaume Sébire}

\footnotetext{
ADRESSE

M. Tardieu : professeur des universités, praticien hospitalier. S. Peudenier : interne des hôpitaux de Rennes, boursière ANRS. G. Sébire : interne des hôpitaux de Paris. Laboratoire de neurovirologie Inserm U. 56, hôpital Bicêtre, 94275 Le Kremlin-Bicêtre Cedex, France.

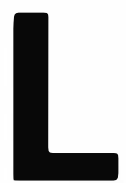

e cerveau doit pouvoir se déf endre contre les agressions extérieures. L'extrême importance de cet organe devrait avoir induit, au cours de l'évolution, la mise en place d'un grand nombre de moyens de protection contre les infections, contre l'inflammation ou encore contre l'auto-immunité. Ces moyens de protection ont longtemps été mal connus, et le cerveau paraissait un organe à part, un site "privilégié ". Mais est-ce vraiment un privilège que de ne pas avoir de circuit lymphatique, d'avoir un endothélium vasculaire non fenêtré qui limite considérablement l'entrée des lymphocytes, de ne pas exprimer les antigènes des complexes majeurs d'histocompatibilité à la surface de ses principales cellules, comme les neurones ou les astrocytes ? Cette notion de site privilégié a dû être totalement reconsidérée à la lumière de données nouvelles, pour certaines encore en discussion [1-3]. Les lymphocytes, tout d'abord, ont la possibilité de passer la barrière hémato-encéphalique à la condition d'avoir été préstimulés : il a été démontré, chez l'animal, que le cerveau est patrouillé en permanence par des lymphocytes activés. Autre donnée nouvelle, le rôle de cellule présentatrice d'antigène peut être exercé par deux cellules du cerveau, l'astrocyte et surtout la cellule microgliale [2-4]. La meilleure connaissance de la cellule microgliale humaine, et de son rôle central dans la réponse immune intracérébrale, est un dernier apport important [2-4].

La description des macrophages résidents du cerveau, formant la microglie, n'est pas d'une très grande nouveauté, puisqu'elle date de 1932, à 


\section{RÉFÉRENCES}

1. Wekerle $\mathrm{H}$, Linington $\mathrm{C}$, Lassmann $\mathrm{H}$, Meyermann R. Cellular immune reactivity within the CNS. Trends Neurosci 1986; 9 : 271-7.

2. Lipkin WI, Tyler KL, Waksman BH. Viruses, the immune system and the central nervous diseases. Trends Neurosci 1988 ; $11: 43-5$.

3. Tardieu M, Boespflug O. Infections virales persistantes du cerveau de l'enfant. médecine/sciences $1988 ; 4: 560-7$.

4. Perry VH, Gordon S. Macrophages and microglia in the nervous system. Trends Neurosci $1988 ; 11: 273-7$.

5. Perry VH, Hume DA, Gordon S. Immunohistochemical localization of macrophages and microglia in the adult and developing mouse brain. Neuroscience $1985 ; 15$ : 313-26.

6. Streit WJ, Graeber MB, Kreutzberg GW. Functional plasticity of microglia : a review. Glia 1988; 1: 301-7.

7. Hickey WF, Kimura H. Perivascular microglial cells of the CNS are bone marrow-derived and present antigen in vivo. Science $1988 ; 239: 290-2$.

8. Choi BH. Hematogenous cells in the central nervous system of developing human embryos and fetuses. J Comp Neurol 1981; $196: 683-94$

9. Ling EA, Penney D, Leblond CP. Use of carbon labeling to demonstrate the role of blood monocytes as precursors of the " ameboid cells" present in the corpus callosum of postnatal rats. J Comp Neurol 1980 ; 193: 631-57.

10. Giulian D, Baker TJ, Shih LN, Lachman LB. Interleukin 1 of the central nervous system is produced by ameboid microglia. J Exp Med 1986 ; 164 : 594-604.

11. Peudenier S, Hery C, Montagnier L, Tardieu M. Human microglial cells : characterization in cerebral tissue and in primary culture, and study of their susceptibility to HIV1 infection. Ann Neurol 1991 . 29: $152-60$.

12. Peudenier S, Hery C, Ng KH, Tardieu M. HIV receptor within the brain : study of CD4 and MHCII on human neurons, astrocytes and microglial cells. Res Virol 1991; 142 : 145-9.

13. Dubois JH, Hammond-Tooke GD, Cuzner L. Expression of major histocompatibility complex antigens in neonate rat primary mixed glial cultures. J Neuroimmunol $1985 ; 9$ : 363-77.

14. Frei K, Siepl C, Groscurth P, et al. Antigen presentation and tumor cytotoxicity by interferon-g-treated microglial cells. Eur J Immunol 1987 ; 17 : 1271-8.

15. Mauerhoff T, Pujol-Borrell R, Mirakian R, Bottazzo GF. Differential expression and regulation of major histocompatibility complex (MHC) products in neural and glial cells of the human fetal brain. $J$ Neuroimmunol $1988 ; 18$ : 271-89.

16. Selmaj KW, Farooq M, Norton WT, et al. Proliferation of astrocytes in vitro in response to cytokines, a primary role for tumor necrosis factor. J Immunol 1990 ; 144: 129-35. une époque où del Rio Hortega ne pouvait utiliser que des colorants tissulaires, en particulier argentiques, pour en démontrer l'existence. Cette limitation technique a masqué l'importance de cette découverte, et ce n'est que récemment, grâce aux techniques de culture et de marquage in vitro, que les nombreux rôles fonctionnels des macrophages du cerveau humain commencent à se dévoiler. Le fait que ces macrophages soient, dans le cerveau, la cible principale de l'infection par le virus de l'immunodéficience humaine, et donc le relais obligatoire des anomalies cérébrales au cours du SIDA, a brutalement jeté une lumière différente sur la recherche dans ce domaine.

Les macrophages sont très abondants dans le cerveau humain normal. Ils semblent constituer $20 \%$ des cellules gliales, ce que l'on peut apprécier en utilisant un marqueur de membrane spécifique des macrophages, l'antigène $\mathrm{Ki}-\mathrm{M} 7$. Cette fréquence est égale dans les différentes parties du cerveau, en particulier le cortex (c'est-à-dire la couche externe du cerveau comportant les neurones) et la substance blanche (c'est-à-dire la zone plus interne de passage des fibres myélinisées) [4-6]. Mais, plus encore que le nombre des cellules macrophagiques importe l'étendue de leur membrane : ces cellules ont de très longs prolongements membranai- res qui s'infiltrent dans le tissu cérébral et augmentent ainsi les possibilités de contacts avec les autres types de cellules du cerveau.

\section{Microglie résidente, microglie périvasculaire et monocytes : filiation ou lointain cousinage?}

Il existe plusieurs types de macrophages dans le cerveau, décrits initialement d'après leur morphologie sur coupe de tissu, puis par leurs fonctions et leurs antigènes de membrane lorsqu'ils ont pu être obtenus ex vivo, à partir de tissus embryonnaires ou de biopsies cérébrales.

La microglie résidente, le premier type de macrophage du cerveau, peut avoir deux formes : ramifiée, qui est probablement celle des macrophages les plus matures, et améboïde, surtout présente au cours du développement embryonnaire et après lésion cérébrale (figure 1) [4]. Un autre type de cellules microgliales a été décrit autour des vaisseaux et est dit ainsi microglie périvasculaire [7]. Le monocyte, issu du sang circulant, est probablement un troisième type de macrophage présent dans le cerveau, mais cette question a soulevé un long débat, qui n'est pas totalement clos. Le passage dans le cerveau normal du monocyte à travers l'endothélium vasculaire, formant la barrière

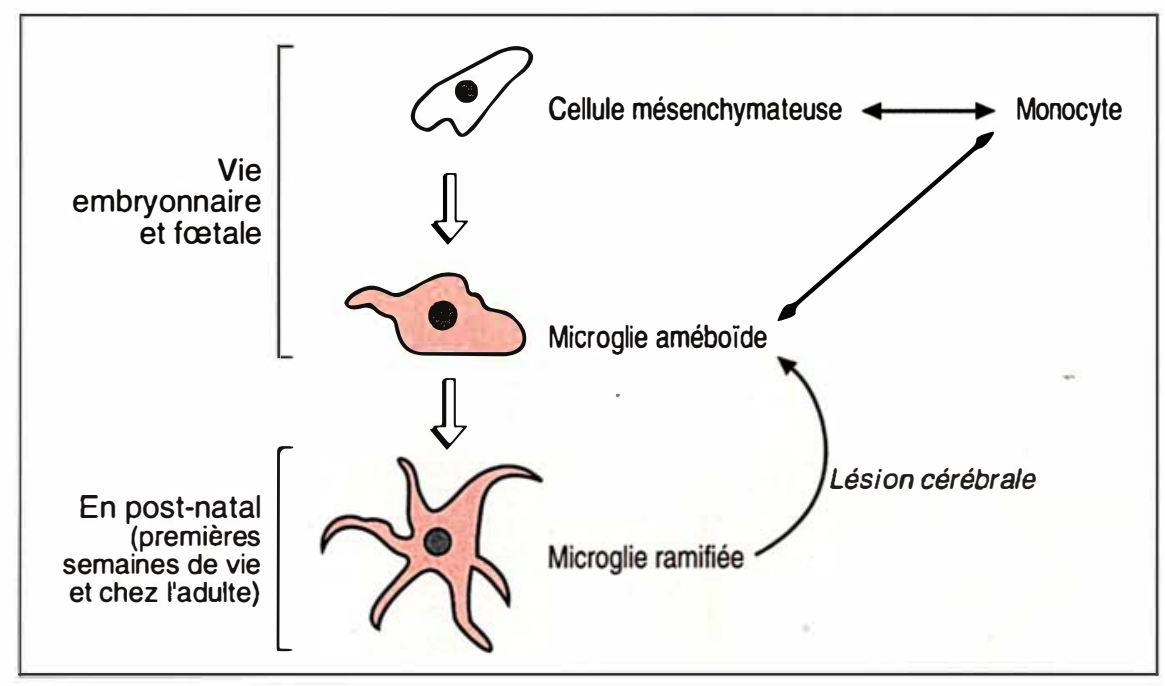

Figure 1. Schéma de l'évolution des macrophages du cerveau du monocyte à la microglie. 


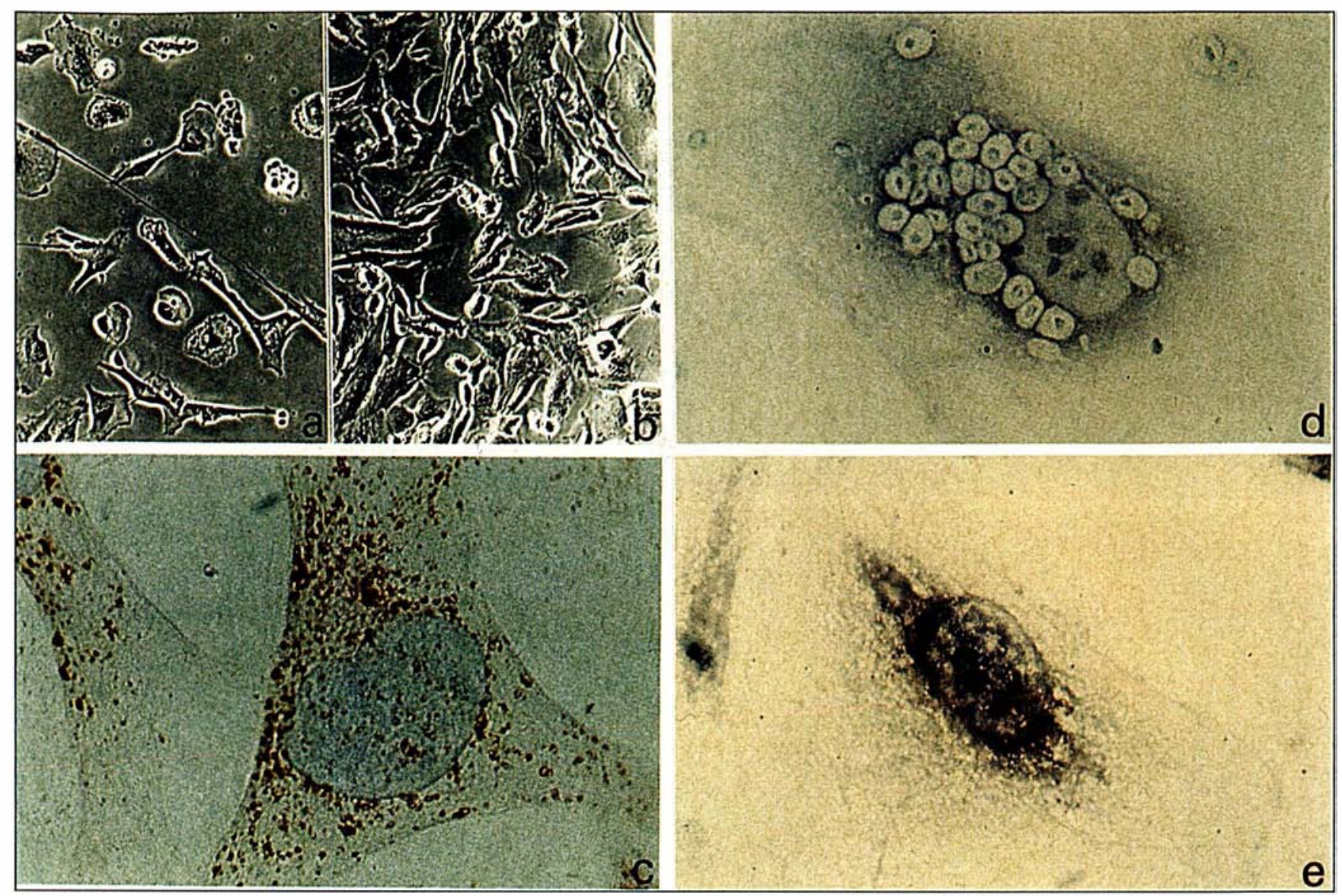

Figure 2. Microglie embryonnaire humaine en culture : aspect morphologique au $4^{\theta}$ et $7^{\theta}$ jour en culture la et b) et démonstration de trois propriétés caractéristiques : expression de l'antigène Ki-M7 (c), capacité de phagocytose (particules de zymosan) (d) et présence d'une activité estérase non spécifique (e).

hémato-encéphalique, n'est pas tout à fait démontré [7-9]. Il semble dépendre de l'expression, à la surface des monocytes, de certains antigènes - et en particulier de certaines protéines d'adhérence qui permettent une liaison entre le monocyte et les cellules de l'endothélium vasculaire. A l'inverse, les cellules microgliales ou les monocytes, par l'intermédiaire de cytokines qu'ils sécrètent, peuvent influencer la perméabilité de la barrière hémato-encéphalique en induisant l'expression de protéines d'adhérence à la surface des cellules endothéliales [10].

De très nombreux antigènes sont exprimés à la surface et dans le cytoplasme des cellules microgliales observées en culture ou sur coupes de tissu cérébral humain (Tableau I, figures 2 et 3) [11, 12]. Ces antigènes ont pu être comparés à ceux présents à $m / s n^{\circ} 8$, vol. 7 , oclobre 91

\begin{tabular}{|lrl|ll|}
\hline \multicolumn{5}{|c|}{ Tableau I } \\
ANTIGĖNES DE SURFACE OU INTRA-CYTOPLASMIQUES \\
CARACTÉRISANT LA MICROGLIE EX VIVO ET IN VIVO \\
\hline \multicolumn{3}{|c|}{ \% Cellules positives } & Présence de cellules marquées \\
\hline Antigène testé & Microglie & Monocyte & Tissu cérébral & Infection HIV-1 \\
& & & normal & \\
\hline Récepteur Fc1 & 77 & 82 & +++ & +++ \\
Récepteur CR32 & 88 & 89 & NF 4 & NF \\
Ki-M7 & 83 & 85 & +++ & +++ \\
Ki-M6 & 18 & 76 & Périvasculaire 5 & ++ \\
CD14 & 34 & 61 & Périvasculaire & ++ \\
CD4 & $<1$ & 82 & Périvasculaire & ++ \\
CD11c & 26 & 85 & ++ & ++ \\
EBM11 & 33 & 50 & +++ & +++ \\
MHCI13 & 4 & 85 & ++ & ++ \\
\hline
\end{tabular}

1 : récepteur pour la fraction Fc des immunoglobulines.

2 : récepteur pour la $3^{0}$ fraction du complément.

3 : antigènes du complexe majeur d'histocompatibilité de type II.

4: $N F=$ non fait.

5 : l'expression de ces antigènes est faible et uniquement périvasculaire (figure 2 ). 

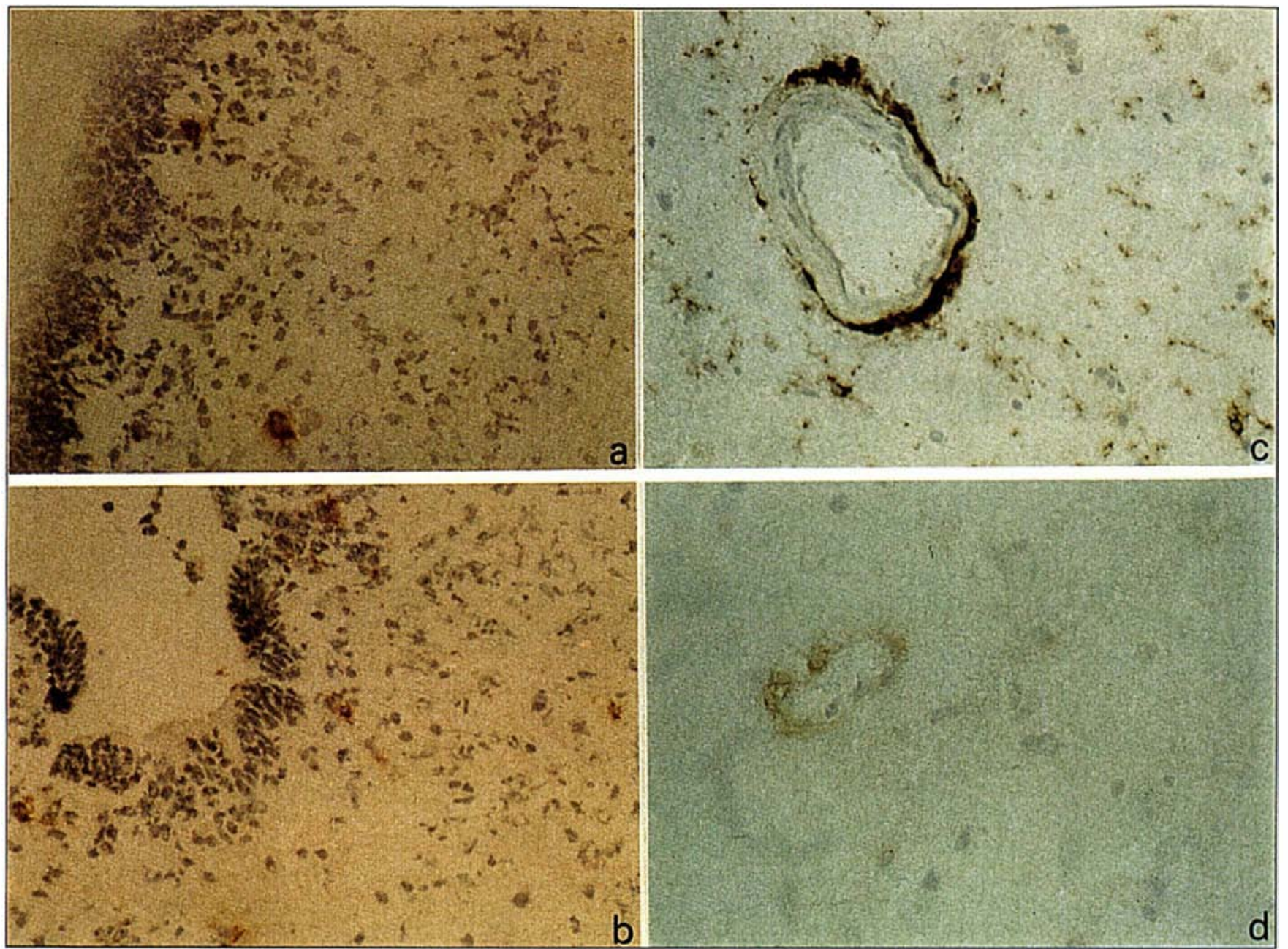

Figure 3. Détection de marqueurs macrophagiques dans le tissu cérébral embryonnaire et adulte. a et $b$ : expression de l'antigène Ki-M7/CD68 dans le tissu nerveux (cortex : a ; moelle : b) d'embryon humain de 10 semaines. Des cellules macrophagiques ont déjà envahi le tissu cérébral. $\boldsymbol{c}$ et $\boldsymbol{d}$ : expression de l'antigène Ki-M7 (marqueurs de microglie tissulaire; photo c) et de l'antigène Ki-M6 (marqueurs des monocytes et de la microglie périvasculaire uniquement; photo d) dans le tissu cérébral adulte normal. Deux types de macrophages existent dans le cerveau : la microglie tissulaire et la microglie périvasculaire très proche, antigéniquement, des monocytes.

la surface du monocyte isolé du sang périphérique. Certains antigènes sont communs aux trois types de macrophages du cerveau, d'autres permettent de les distinguer. Les monocytes et les cellules microgliales périvasculaires partagent de très nombreux antigènes; au contraire, les cellules microgliales tissulaires n'expriment plus certains antigènes, comme le montre le Tableau I, et cela est en particulier vrai pour la molécule CD4. Cela suggère une filiation entre les trois types de cellules, sans démontrer formellement le passage d'une forme à l'autre, et tout parti- culièrement la transformation du monocyte en microglie périvasculaire.

\section{Les modes d'action de la microglie}

Les cellules microgliales possèdent toutes les propriétés des macrophages tant in vivo qu'ex vivo, avec cependant des variations d'expression en fonction de la maturation de la cellule. Elles ont trois rôles de grande importance : la phagocytose, la présentation d'antigènes étrangers aux lymphocytes et la sécrétion de cytokines.
La microglie possède plusieurs voies pour internaliser des particules ou des antigènes étrangers. Elle peut les phagocyter simplement, comme le montre la figure 2 pour des particules de zymosan : il est très vraisemblable que l'internalisation de nombreuses particules bactériennes ou virales passe par cette voie. La capacité de phagocytose est cependant d'autant plus importante que la cellule est plus jeune et semble être très réduite dans la microglie très mûre, au moins ex vivo. La cellule microgliale peut également utiliser ses récepteurs pour la fraction $\mathrm{Fc}$ des 
immunoglobulines ou ses récepteurs pour la fraction C3 du complément pour internaliser des complexes immuns, c'est-à-dire des structures antigéniques recouvertes d'anticorps [11]. A l'intérieur du cytoplasme des cellules microgliales s'expriment de nombreuses activités enzymatiques comme l'activité estérase non spécifique, que possèdent la plupart des cellules macrophagiques (figure 2). De plus, les cellules macrophagiques nouvellement recrutées ont une grande capacité à engendrer des métabolites de l'oxygène, très cytotoxiques, qui ont un rôle important dans la défense contre l'infection [4]. La présentation d'antigènes étrangers intracérébraux aux lymphocytes patrouillant le tissu cérébral, nécessite la présence des antigènes du complexe majeur d'histocompatibilité de type II. Ces antigènes, assez largement exprimés à la surface du monocyte, ont, ex vivo, une faible expression spontanée à la surface de la cellule microgliale. Cependant, leur expression peut être activée par de nombreux facteurs comme les cytokines, en particulier l'interféron gamma, ou les lipopolysaccharides présents dans de très nombreux germes [11-15].

La microglie est probablement la principale cellule sécrétrice de cytokines du système nerveux central humain. Ainsi avons-nous observé récemment qu'elle est, ex vivo, la principale, si ce n'est la seule, cellule sécrétrice d'IL-6. Elle semble également pouvoir sécréter, mais dans une bien moindre mesure que l'IL-6, de l'IL-1 ou du TNF (tumor necrosis factor). La sécrétion de cytokines est autocrine car la microglie peut être aussi cible des cytokines. Il est très probable que la libération de cytokines se fasse non seulement sous forme de facteurs solubles circulants, mais aussi au niveau de sites d'adhérence entre la cellule microgliale et les astrocytes ou les neurones. Il existe une étroite interaction entre un astrocyte et une cellule microgliale $e x$ vivo, mais l'expression des protéines d'adhérence à la surface de la microglie est encore mal connue. Les cytokines, dans le cerveau, ont des fonctions multiples. Elles agissent surtout dans l'élaboration et l'amplification de la réponse immune locale, mais aussi dans la prolifération astro- cytaire, l'angiogenèse et la différentiation neuronale $[10,16]$.

\section{La cellule microgliale au cours de la formation du cerveau}

Au cours de la formation du cerveau, de nombreuses morts cellulaires sont programmées. Il est très vraisemblable que la microglie ait un rôle important dans ce processus, mais cela n'est vraiment bien connu que chez l'animal. Un autre article de cette revue aborde cette question. Tenter de déterminer quand la microglie s'installe dans le cerveau humain en voie de formation, quand la transition du monocyte à la microglie s'y opère, est cependant de grand intérêt. Une série d'expériences récentes nous ont permis d'observer la présence de nombreux antigènes caractéristiques de macrophages dans le système nerveux d'embryons humains dès la $8^{e}$ semaine (figure 3) [11]. Microglies matures et monocytes, reconnus par leur différents antigènes de membranes, cohabitent encore dans l'ensemble du tissu cérébral comme si la transition du monocyte à la cellule microgliale tissulaire n'était pas achevée. Ces cellules microgliales et monocytaires sont présentes à la partie externe de la zone périventriculaire de prolifération neuronale, à la base des colonnes de migration (figure 3). De tels résultats viennent appuyer l'étude morphologique plus ancienne de Choi qui avait observé la présence de nombreuses cellules hématogènes sur des coupes de système nerveux d'embryons humains dès 6 semaines. Ces cellules lui paraissaient être moins fréquemment observées après la $15^{\mathrm{e}}$ semaine [8]. Des macrophages sont donc présents très tôt dans le système nerveux central humain en développement. Leurs multiples rôles, comme, par exemple, la sécrétion de facteurs de croissance ou la régulation de la mort neuronale programmée, restent encore à démontrer chez l'homme.

\section{La microglie en pathologie humaine}

- Microglie, cellule cible d'infections : l'exemple du SIDA

Dans de nombreuses affections, le germe infectant a une affinité particulière pour les macrophages. C'est le cas, par exemple, du bacille de Koch ou de la Listeria. Le virus de l'immunodéficience humaine de type 1 (HIV-1), cause du SIDA, est cependant le germe dont les rapports avec la microglie et l'ensemble du système macrophagique intracérébral est actuellement le plus étudié.

Le HIV-1 est capable d'infecter in vivo et ex vivo certains lymphocytes et monocytes après fixation du virus sur son principal récepteur, la molécule CD4. Cette molécule est plus abondamment exprimée sur les lymphocytes que sur les monocytes, ce qui rend compte, en partie seulement, de la différence de capacité à répliquer le virus entre ces deux cellules. Dans le cerveau de sujets adultes décédés de SIDA, les antigènes du HIV-1 ne peuvent être retrouvés que dans des macrophages [17-20]. Les neurones et les astrocytes, tant ex vivo qu'in vivo, semblent tout à fait résistants à l'infection par HIV-1, sauf si des conditions expérimentales très particulières sont utilisées (utilisation de très haut titre de virus ou transfection) ([21-24] et M. Tardieu et al., soumis pour publication).

Si la seule cellule intracérébrale infectée par le HIV-1 est de type macrophage, il est très important de déterminer si elle est un monocyte ayant passé la barrière hémato-encéphalique ou une cellule microgliale tissulaire mûre, car les modalités de lutte contre l'infection et ses conséquences pourraient différer dans chacun de ces cas. Ainsi, par exemple, si on peut démontrer que les cellules infectées sont uniquement des monocytes qui passent la barrière hématoencéphalique, il pourrait être possible de lutter contre ce passage et de limiter la pénétration intracérébrale de cellules infectées. Plusieurs arguments, issus d'un travail récent de notre équipe, suggèrent, en effet, que les macrophages infectés dans le cerveau sont surtout des monocytes. Le premier argument a été obtenu lors de l'examen de coupes de cerveau de sujets décédés de SIDA. Sur ces coupes tissulaires, les monocytes (reconnus par leurs antigènes de membrane) sont retrouvés dans l'ensemble du parenchyme cérébral et non uniquement en situation périvascu- 


\section{RÉFÉRENCES}

17. Koenig S, Gendelman $\mathrm{H}$, Orenstein JM, et al. Detection of AIDS virus in macrophages in brain tissue from AIDS patients with encephalopathy. Science 1986 233 : 1089-93.

18. Price RW, Brew B, Sidtis J, et al. The brain in AIDS : central nervous system HIV1 infection and AIDS dementia complex. Science 1988 ; 239 : 586-92.

19. Vazeux R, Brousse N, Jarry A, et al. AIDS subacute encephalitis : identification of HIV-infected cells. Am J Pathol 1987 ; 126 : 403-9.

20. Wiley CA, Schrier RD, Nelson JA, $e$ al. Cellular localization of human immunodeficiency virus infection within the brain of acquired immune deficiency syndrome patients. Proc Natl Acad Sci USA 1986 ; 83 7089-93.

21. Chesebro B, Buller R, Portis J, Wehrly K. Failure of human immunodeficiency virus entry and infection in CD4-positive human brain and skin cells. $J$ Virol $1990 ; 64: 215-21$.

22. Chiodi F, Fuerstenberg S, Fenyö EM, et al. Infection of brain-derived cells with the human immunodeficiency virus. $J$ Virol 1987 ; $61: 1244-7$.

23. Cheng-Mayer C, Tutka JT, Levy JA, et al. Human immunodeficiency virus can productively infect cultured human glial cells. Proc Natl Acad Sci USA 1987 ; 84 3526-30.

24. Dewhurst S, Sakai K, Volsky DJ, et al. Persistent productive infection of human glial cells by human immunodeficiency virus (HIV) and by infectious molecular clones of HIV. J Virol 1987 ; 61 : 3774-82.

25. Watkins BA, Dorn HH, Kelly WB, $e^{t}$ al. Specific tropism of HIV1 for microglial cells in primary human brain cultures Science 1990 ; 249 : 549-53.

26. Jordan CA, Watkins BA, Kufta C Dubois-Dalcq $M$. Infection of brain microglial cells by human immunodeficiency virus type 1 is CD4 dependent. J Virol 1991; 65 : 736-42.

27. Dreyer EB, Kaiser PK, Offermann JT, Lipton SA. HIV1 coat protein neurotoxicity prevented by calcium channel antagonists. Science $1990 ; 248$ : 364-7.

28. Kaiser PK, Offermann JT, Lipton SA. Neuronal injury due to HIV1 envelope protein is blocked by anti-gp120 antibodies but not by anti-CD4 antibodies. Neurology 1990 ; 40 : 1757-61

29. Sabatier JM, Vives E, Mabrouk K, $e$ al. Evidence for neurotoxic activity of tat from human immunodeficiency virus type 1 . Jirol 1991 ; 65 : 961-7.

30. Giullian D, Vaca K, Noonan CA Secretion of neurotoxins by mononuclear phagocytes infected with HIV1. Science 1990 ; 250 : 1593-6.

31. Hofman FM. Cytokines in central nervous system diseases. In : Goetzl EJ, Spector NH, eds. Neuroimmune Networks : Physiology and Diseases. New York: Alan Riss Inc, laire comme dans le cerveau de sujets adultes non infectés (Tableau I et [11]). De plus, les cellules contenant des antigènes viraux (les antigènes p18 et p24) expriment aussi, en double marquage, des antigènes de membrane de type monocytaire. D'autres informations sont venues d'expériences ex vivo. Les cellules microgliales en culture n'expriment pas le récepteur habituel de HIV-1, la molécule CD4 : l'antigène ne peut être mis en évidence par immunomarquage, ni l'ARN CD4, par northern blot. De plus, entre nos mains, les cellules microgliales mûres, infectées ex vivo par HIV-1, ne montrent aucun signe direct ou indirect de réplication virale, que l'infection soit faite sur des cellules au repos ou après stimulation par des cytokines ou du LPS [11]. Il est cependant possible que d'autres modes de stimulation, voire certaines co-infections, permettent de réaliser une telle infection. Un travail récent d'une autre équipe, testant des macrophages issus d'une tumeur gliale, a permis d'obtenir une réplication virale après stimulation des cellules par un ensemble de cytokines ou facteurs de croissance $[25,26]$. Cependant, l'origine monocytaire ou microgliale des macrophages testés n'est que très peu analysée dans ce travail.
On peut donc faire l'hypothèse raisonnable que la principale cellule infectée par HIV-1 dans le cerveau est de type monocytaire. Il peut s'agir soit de monocytes ayant passé la barrière hémato-méningée, soit de cellules de la microglie périvasculaire, très proches antigéniquement des monocytes, qui pourraient phagocyter, après leur traversée de l'endothélium vasculaire, des particules virales ou des lymphocytes infectés. Deux mécanismes, au moins, pourraient expliquer comment les macrophages infectés agissent, à l'intérieur du système nerveux central, sur les neurones et les astrocytes. Les macrophages peuvent sécréter des facteurs solubles qui pourraient agir à distance sur ces cellules. Ils peuvent aussi adhérer directement à la surface des cellules du système nerveux central et modifier ainsi les membranes cellulaires. Une série d'articles très récents a apporté des débuts de réponse. Deux des protéines du virus, la gp120 et la protéine Tat, peuvent modifier la survie de neurones et d'astrocytes [27-29]. Cela n'a cependant été étudié encore que chez le rongeur. La gp120 exerce son action en modifiant les canaux calcium, tandis que Tat agit par une autre voie, en modifiant la polarisa. tion membranaire $[28,30]$. Dans un

\begin{tabular}{|c|c|c|c|c|}
\hline \multicolumn{5}{|c|}{$\begin{array}{l}\text { Tableau II } \\
\text { V D'IL-6, TNF et IL-1 } \\
\text { ES PATHOLOGIES NEUROLOGIQUES, } \\
\text { R COUPE DE TISSU CÉRÉBRAL }\end{array}$} \\
\hline \multirow{2}{*}{ Maladies neurologiques } & \multirow{2}{*}{ Localisation } & \multicolumn{3}{|c|}{ Détection de cytokines } \\
\hline & & IL-6 & TNF & IL-1 \\
\hline $\begin{array}{l}\text { Méningites bactériennes } \\
\text { Méningites virales } \\
\text { Encéphalite herpétique } \\
\text { Myélite due à HTLV1 } \\
\text { SIDA: } \\
\text { - sans signe neurologique } \\
\text { - syndrome démentiel } \\
\text { - infections opportunistes } \\
\text { Panencéphalite } \\
\text { sclérosante subaiguë } \\
\text { Sclérose en plaques } \\
\text { Neurolupus } \\
\text { Maladie d'Alzheimer }\end{array}$ & $\begin{array}{l}\text { LCR } \\
\text { LCR } \\
\text { LCR } \\
\text { LCR } \\
\text { LCR } \\
\text { LCR } \\
\text { LCR } \\
\text { Portex } \\
\text { LCR } \\
\text { Cortex } \\
\text { LCR } \\
\text { LCR } \\
\text { Cortex } \\
\text { LCR }\end{array}$ & $\begin{array}{l}++ \\
+ \\
+ \\
+ \\
- \\
++ \\
++ \\
+ \\
+ \\
+ \\
++ \\
? \\
+\end{array}$ & $\begin{array}{l}+++ \\
- \\
? \\
? \\
- \\
- \\
+ \\
+ \\
+ \\
+ \\
? \\
? \\
?\end{array}$ & $\begin{array}{l}+++ \\
+ \\
? \\
? \\
+ \\
++ \\
++ \\
++ \\
? \\
? \\
? \\
? \\
? \\
++ \\
?\end{array}$ \\
\hline
\end{tabular}


autre travail, il a été démontré qu'un autre facteur soluble, sécrété par des macrophages infectés par HIV-1, provoque la mort cellulaire de neurones de rat et de poulet, en modifiant non pas les canaux calcium, mais un récepteur membranaire tout à fait essentiel à la surface des neurones, le récepteur N-méthyl-Daspartate (NMDA) [30]. La nature de ce facteur est encore inconnue, mais il ne semble s'agir ni de peptides viraux, ni de radicaux libres, ni de cytokines. A côté de ces travaux concernant des facteurs solubles, nous avons observé qu'une adhérence des monocytes aux neurones et astrocytes humains était également possible et pouvait représenter un autre mode d'induction de lésions neuronales et astrocytaires (M. Tardieu et al., soumis pour publication). Des cellules d'une lignée continue monocytoïde (cellules U 937), infectées ou non par HIV-1, ont été co-cultivées avec des neurones et astrocytes humains. L'adhérence débute après quelques jours en culture et est beaucoup plus fréquente et rapide pour les cellules inf ectées que pour les cellules qui ne le sont pas. Autour de la cellule macrophagique infectée et au moment où celle-ci réplique le virus, de larges plaques de nécrose se forment. Ici aussi, le mécanisme de la nécrose paraît dépendre de la diffusion d'antigènes viraux au niveau du site d'adhérence et non de la sécrétion de radicaux libres ou de cytokines (M. Tardieu et al., soumis pour publication). Beaucoup de travail reste néanmoins à faire pour clarifier les modalités de l'interaction entre les macrophages du cerveau infectés par HIV-1 et les autres cellules du système nerveux central.

\section{- Microglie, cytokines et patho-} logie inflammatoires du SNC

La sécrétion de cytokines est démontrée dans de nombreuses maladies inflammatoires du cerveau, soit par dosage dans le sérum ou le liquide céphalo-rachidien, soit par immunocytochimie sur coupe de cerveau. Les principaux résultats sont résumés dans le Tableau II [31]. Il faut cependant noter que la détection de ces cytokines au cours d'une affection donnée n'implique pas nécessairement leur rôle comme cause de cette $\mathrm{m} / \mathrm{s} n^{\circ} 8$, col. 7 , octobre 91 affection. La sécrétion de cytokines peut ne faire qu'accompagner le phénomène causal ou, plus vraisemblablement, l'amplifier. Un rôle direct des cytokines paraît très probable au cours de la sclérose en plaques, maladie inflammatoire liée à la destruction par plaque de la myéline cérébrale. La présence de TNF est détectée en immunocytochimie en bordure des plaques de démyélinisation et, ex vivo, le TNF paraît toxique pour la myéline et les oligodendrocytes qui la forment. De même, une sécrétion d'IL-1 et de TNF est démontrée au cours des méningites bactériennes. Ces deux cytokines pourraient agir sur l'inflammation méningée et sur les modifications de la barrière hémato-encéphalique.

- La microglie dans d'autres maladies du cerveau

Il est vraisemblable que d'autres affections cérébrales impliquent la microglie. On peut par exemple penser aux maladies dites de surcharge où, du fait d'une anomalie congénitale dans une chaîne métabolique, se forme une accumulation d'un substrat, le plus souvent lipidique. Les lysosomes des macrophages sont tout particulièrement surchargés, y compris dans le tissu cérébral. Actuellement, le rôle fonctionnel et éventuellement pathogène de la microglie au cours de ces affections est tout à fait mal connu.

\section{Conclusion}

Le cerveau humain contient plusieurs types de macrophages : la microglie tissulaire peut être distinguée de la microglie périvasculaire et des monocytes. La cellule microgliale humaine commence à être mieux définie, grâce à la connaissance que l'on en avait eu précédemment en expérimentation animale et aux possibilités d'isolement et de culture de ces cellules à partir d'échantillons d'origine humaine. Son rôle en pathologie humaine est certainement plus vaste que ce qui est actuellement connu

\section{Remerciements}

Ce travail n'aurait pu être fait sans l'aide technique constante de C. Hery.

\section{Summary}

Macrophages in human brain : from the silver stain to AIDS

Macrophage's arc abundant in the normal adult brain and account for 20 to $30 \%$ of the glial cells. Three types of human brain macrophages can be defined in tissue section or in culture : tissular resident microglial cells, perivascular microglial cells and monocytes. Several antigenic markers including the Fc receptor and the CR3 receptor are common to the three types of brain macrophages whereas other markers, such as CD4 antigens, are expressed on monocytes and perivascular microglial cells but not on tissular resident microglial cells. Microglial cells have important functions in the intracerebral immune response: phagocytosis, antigenic presentation to lymphocytes, secretion of cytokines. These different functions can also be demonstrated on isolated and cultured human microglial cells.

Macrophages are the main infected cells in the brain of patients infected with HIV1. Two series of results suggest that these infected macrophages are monocytes and not the resident microglial cells. First, there is an ex vivo difference in HIV1 susceptibility between monocytes and microglial cells : after stimulation with $\mathrm{Gm}-\mathrm{CSF}$ or TNF, monocytes but not microglial cells can replicate HIV1. Secondly, in the brain of HIV 1 infected patients, infected cells expressed antigens characteristics of monocytes. This suggests that HIV1-infected cells within the brain are either monocytes which would have crossed the bloodbrain-barrier after their infection with HIV1 or perivascular microglial cells which, after phagocyting infected blood lymphocytes or free viral particles, would subsequently contain viral antigen and migrate into brain tissue.

\section{TIRÉS A PART}

M. Tardieu. 